\title{
reciamuc
}

Revista cientifica de investigación actualización del mundo de las ciencias

\author{
Victoria Samanta Luna Panimboza ${ }^{\text {a }}$; Kristell Solange Ladines Torres ${ }^{\text {b }}$; Carlos \\ Emilio Paz Illescas ${ }^{c}$; Isrrael Rubén Rugel Cabrera ${ }^{\mathrm{d}}$
}

Enfermedad celíaca en síndrome de Down

Celiac disease in Down syndrome

Revista Científica de Investigación actualización del mundo de las Ciencias. Vol. 3 núm., 1, enero, ISSN: 2588-0748, 2018, pp. 570-586

DOI: $10.26820 /$ reciamuc/3.(1).enero.2019.570-586

URL: http://reciamuc.com/index.php/RECIAMUC/article/view/248

Código UNESCO: 3205 Medicina Interna

Tipo de Investigación: Artículo de Revisión

Editorial Saberes del Conocimiento

Recibido: 10/12/2018

Aceptado: 20/01/2019

Publicado: 30/01/2019

Correspondencia: director@ reciamuc.com

a. Médico; Guayaquil, Ecuador.
b. Médico; Guayaquil, Ecuador.
c. Médico; Guayaquil, Ecuador.
d. Médico; Guayaquil, Ecuador. 


\section{Enfermedad celíaca en síndrome de Down}

Vol. 3, núm. 1., (2019)

Victoria Samanta Luna Panimboza; Kristell Solange Ladines Torres; Carlos Emilio Paz Illescas;

Isrrael Rubén Rugel Cabrera

\section{RESUMEN}

La enfermedad celíaca es la intolerancia total y permanente a proteínas (prolaminas) presentes en el trigo, la avena, la cebada y el centeno que ocasiona atrofia vellositaria de la mucosa del intestino delgado ocasionando una deficitaria absorción de nutrientes. Se induce por la ingesta de gluten, que es una proteína rica en residuos de glutamina y prolina. La gliadina es la fracción soluble en alcohol del gluten y contiene la mayor parte de los productos tóxicos. Estos péptidos atraviesan la barrera epitelial del intestino cuando hay un aumento de la permeabilidad en esa zona, al reaccionar con las células presentes desencadena una respuesta inflamatoria medida por linfocitos $\mathrm{T}$ helper tipo 1. Las personas con síndrome de Down presentan ciertos riesgos y problemas de salud propios de este síndrome que requieren acciones precisas para garantizar el pleno desarrollo y aprovechamiento de las capacidades de cada persona con esta condición. El mayor conocimiento de los riesgos asociados a este síndrome permite conocer qué alteraciones pueden aparecer y en qué momento o etapa de la vida del paciente, siendo posible añadir un grupo de actividades preventivas así como exploraciones que buscan corregir, aliviar o evitar problemas de salud de estos pacientes. La historia del paciente y el examen clínico constituyen la base para orientar el diagnostico en relación a la atención primaria, la endoscopia de la vía digestiva alta, el gastroenterólogo examinará el intestino y tomará una biopsia para observar si existen signos de enfermedad celíaca. El régimen terapéutico consiste en una dieta estricta sin gluten que deberá mantener el paciente a lo largo de la vida, obteniendo la desaparición de los síntomas clínicos y la normalización de la mucosa intestinal, se evitan las posibles complicaciones tardías derivadas del consumo del gluten. Se excluirá cualquier producto que contenga trigo, avena, cebada, centeno y todos sus derivados de la dieta del paciente celíaco. La metodología usada es descriptiva, con un enfoque documental, es decir, revisar fuentes disponibles en la red, como google académico, con contenido oportuno y relevante desde el punto de vista científico que enriquezca el análisis del tema planteado en este artículo.

Palabras Claves: Gluten; Síndrome Down; Celíaco; Biopsia; Vellosidad Intestinal; Trastorno Genético; DQ2 y DQ8. 


\section{Enfermedad celíaca en síndrome de Down}

Vol. 3, núm. 1., (2019)

Victoria Samanta Luna Panimboza; Kristell Solange Ladines Torres; Carlos Emilio Paz Illescas; Isrrael Rubén Rugel Cabrera

\section{ABSTRACT}

Celiac disease is the total and permanent intolerance to proteins (prolamines) present in wheat, oats, barley and rye that causes villous atrophy of the small intestinal mucosa causing a deficient nutrient absorption. It is induced by the intake of gluten, which is a protein rich in glutamine and proline residues. Gliadin is the alcohol-soluble fraction of gluten and contains most of the toxic products. These peptides cross the epithelial barrier of the intestine when there is an increase in permeability in that area, reacting with the cells present triggers an inflammatory response measured by helper T lymphocytes type 1. People with Down syndrome present certain risks and health problems of this syndrome that require precise actions to guarantee the full development and use of the abilities of each person with this syndrome. The greater knowledge of the risks associated with this condition allows knowing what alterations may appear and at what time or stage of the patient's life, being possible to add a group of preventive activities as well as explorations that seek to correct, alleviate or avoid health problems of these patients. The clinical history and clinical examination are the basis to guide the diagnosis in relation to primary care, endoscopy of the upper digestive tract, the gastroenterologist will examine the intestine and take a biopsy to see if there are signs of celiac disease. The therapeutic regimen consists of a strict gluten-free diet that the patient must maintain throughout his life, obtaining the disappearance of the clinical symptoms and the normalization of the intestinal mucosa, avoiding the possible late complications derived from the consumption of gluten. Any product containing wheat, oats, barley, rye and all its derivatives from the diet of the celiac patient will be excluded. The methodology used is descriptive, with a documentary approach, that is, to review sources available on the web, such as academic google, with timely and relevant content from a scientific point of view that enriches the analysis of the topic raised in this article.

Key Words: Gluten; Down Syndrome; Celiac; Biopsy; Intestinal Villi; Genetic Disorder; DQ2 and DQ8. 


\section{Enfermedad celíaca en síndrome de Down}

Vol. 3, núm. 1., (2019)

Victoria Samanta Luna Panimboza; Kristell Solange Ladines Torres; Carlos Emilio Paz Illescas;

Isrrael Rubén Rugel Cabrera

\section{Introducción.}

Una dieta libre de gluten es algo que está muy de moda, incluso hay personas que sin tener algún impedimento o limitación de salud, decide voluntariamente eliminar el consumo de su dieta de alimentos como: avena, centeno, trigo y todos sus derivados de la dieta del paciente celíaco. Sin embargo, para el paciente diagnosticado con enfermedad celíaca no es una decisión de moda sino de calidad de vida.

La enfermedad celíaca es la intolerancia total y permanente a proteínas (prolaminas) presentes en el trigo, la avena, la cebada y el centeno que ocasiona atrofia vellositaria de la mucosa del intestino delgado ocasionando una deficitaria absorción de nutrientes. Se calcula que aproximadamente 1 de cada 160 la padece y lo ignora porque es asintomática y es mucho más propensa en mujeres que en hombres. (Ali, 2006)

Si adicionalmente el paciente tiene síndrome de Down la enfermedad celíaca puede manifestarse a cualquier edad y tiene una prevalencia mucho mayor que en cualquier otro tipo de población. Se manifiesta más comúnmente como una enfermedad silente, con síntomas vagos como constipación, dispepsia, dolor abdominal y flatulencia. Esta condición dificulta y retarda el diagnóstico en estos pacientes que en la población en general.

La iniciación clínica suele ocurrir en la infancia con la introducción del gluten en la dieta o aparecer tardíamente en la juventud, la edad adulta o la vejez. Por ello se recomienda realizar una evaluación médica a los dos años de vida en los niños con síndrome de Down que descarte la posibilidad de padecerla y en caso de resultar negativo el diagnostico, estar atentos a su posible desarrollo en toda etapa de la vida. 


\section{Enfermedad celíaca en síndrome de Down}

Vol. 3, núm. 1., (2019)

Victoria Samanta Luna Panimboza; Kristell Solange Ladines Torres; Carlos Emilio Paz Illescas; Isrrael Rubén Rugel Cabrera

El propósito de este artículo es concientizar e informar a los lectores de esta enfermedad, estar atentos a los síntomas y/o patologías, a fin de recibir el tratamiento oportuno que mejore la calidad de vida del paciente. Esta información y más disponible a continuación.

\section{Metodología.}

Esta investigación está dirigida al estudio de la enfermedad celíaca en síndrome de Down. Para realizarlo se usó una metodología descriptiva, con un enfoque documental, es decir, revisar fuentes disponibles en la red, como google académico, con contenido oportuno y relevante desde el punto de vista científico para dar respuesta a lo tratado en el presente artículo y que sirvan de inspiración para realizar otros proyectos. Las mismas pueden ser estudiadas al final, en la bibliografía.

\section{Resultados.}

La enfermedad celíaca se defina como un trastorno inmunitario sistémico mediado por el gluten y prolaminas relacionadas en individuos genéticamente predispuesto, caracterizados por la presencia de una combinación de manifestaciones clínicas variables, anticuerpos específicos, haplotipos HLA - DQ2 o HLA - DQ8 y enteropatía. (Díaz, 2012, pág. 28).

Se induce por la ingesta de gluten, que es una proteína rica en residuos de glutamina y prolina. La gliadina es la fracción soluble en alcohol del gluten y contiene la mayor parte de los productos tóxicos. Las moléculas de gliadina no digeridas, tales como la fracción alfa, (contiene 33 aminoácidos) son resistentes a la degradación por el jugo gástrico, pancreático e intestinal. Estos péptidos atraviesan la barrera epitelial del intestino cuando hay un aumento de la 


\section{Enfermedad celíaca en síndrome de Down}

Vol. 3, núm. 1., (2019)

Victoria Samanta Luna Panimboza; Kristell Solange Ladines Torres; Carlos Emilio Paz Illescas;

Isrrael Rubén Rugel Cabrera

permeabilidad en esa zona, al reaccionar con las células presentes desencadena una respuesta inflamatoria medida por linfocitos T helper tipo 1.

Posteriormente se libera una serie de citosinas pro inflamatoria como el interferón gamma y el factor de necrosis tumoral alfa, que contribuye a la producción de la hiperplasia de criptas y desarrollo de la lesión epitelial. Los péptidos de gliadina activa la respuesta inmunitaria innata que origina la estimulación de los linfocitos intraepiteliales que expresa el receptor NK-G2D, un marcador natural de las células citolíticas, estas células activadas destruyen las células del epitelio intestinal (Rodrigo, 2008).

Entre los factores ambientales (además del gluten) que pueden desencadenar la aparición de la enfermedad celíaca hay que destacar la lactancia materna, que retrasa la aparición de la enfermedad, la edad en que se introducen los alimentos con gluten en la dieta del niño, ciertas infecciones intestinales o viajes a países tropicales. El gráfico $\mathrm{N}^{\circ} 1$ ilustra este proceso:

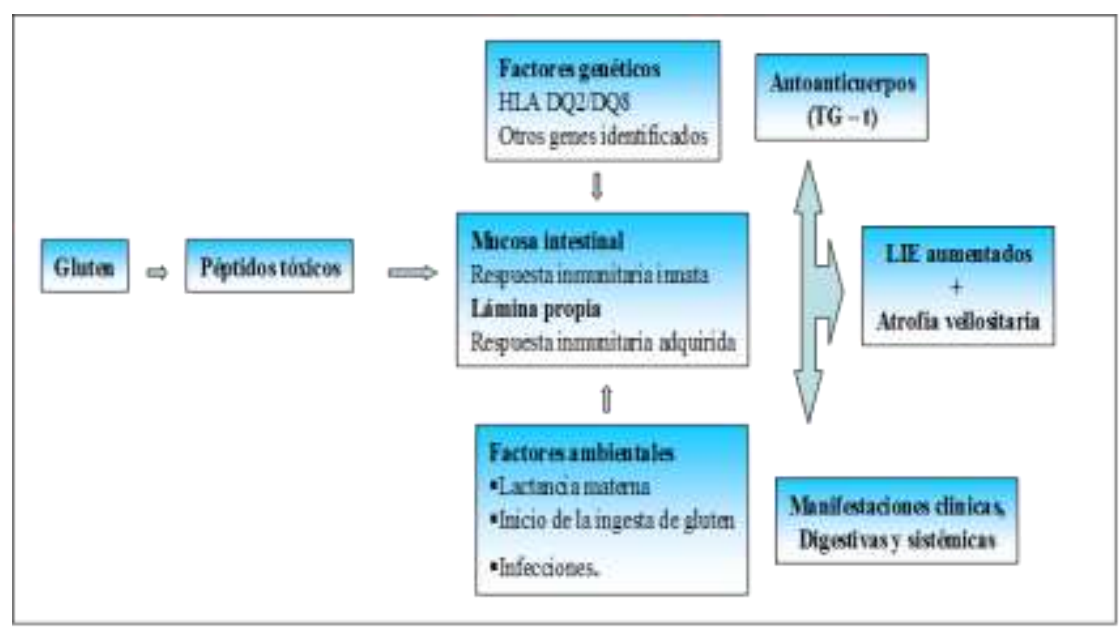

\section{Grafico $N^{\circ}$ 1. Mecanismos patogénicos de la enfermedad celíaca}

Fuente: Rodrigo L. 2008 


\section{Enfermedad celíaca en síndrome de Down}

Vol. 3, núm. 1., (2019)

Victoria Samanta Luna Panimboza; Kristell Solange Ladines Torres; Carlos Emilio Paz Illescas; Isrrael Rubén Rugel Cabrera

Las personas con síndrome de Down presentan ciertos riesgos y problemas de salud propios de este síndrome que requieren acciones precisas para garantizar el pleno desarrollo y aprovechamiento de las capacidades de cada persona con esta condición. El mayor conocimiento de los riesgos asociados a este síndrome permite conocer qué alteraciones pueden aparecer y en qué momento o etapa de la vida del paciente, siendo posible añadir un grupo de actividades preventivas así como exploraciones que buscan corregir, aliviar o evitar problemas de salud de estos niños. La tabla $\mathrm{N}^{\circ} 1$ resume los aspectos más importantes en materia de salud a vigilar durante la atención primaria de estos niños con síndrome de Down.

Tabla $N^{\circ}$ 1. Principales problemas de salud en personas con Síndrome de Down

\section{(Excluida la etapa neonatal)}

\begin{tabular}{|l|c|}
\hline Problema & Prevalencia \\
\hline Cardiopatía congénita & $40-62 \%$ \\
Hipotonía & $100 \%$ \\
Retraso del crecimiento & $100 \%$ \\
Retraso mental & $97.3 \%$ \\
Alteraciones de la audición & $50 \%$ \\
Problemas oculares: & \\
$\quad$ • Errores refracción & $50 \%$ \\
$\quad$ Estrabismo $\quad$ Cataratas & $44 \%$ \\
Anormalidad vertebral cervical & $5 \%$ \\
Alteraciones tiroideas & $10 \%$ \\
Sobrepeso & $45 \%$ \\
Desordenes convulsivos & Común \\
Problemas emocionales y de conducta & $5-10 \%$ \\
Demencia prematura( $5^{\circ}-6^{\circ}$ década) & Común \\
Enfermedad periodontal, caries, malposición & $19-41 \%$ \\
Disgenesia gonadal & $60 \%$ \\
Enfermedad celíaca & $40 \%$ \\
Apnea obstructiva del sueño & $3-7 \%$ \\
\hline
\end{tabular}

Fuente: Soriano J. (2007) 


\section{Enfermedad celíaca en síndrome de Down}

Vol. 3, núm. 1., (2019)

Victoria Samanta Luna Panimboza; Kristell Solange Ladines Torres; Carlos Emilio Paz Illescas;

Isrrael Rubén Rugel Cabrera

Se evidencia en la información mostrada anteriormente que uno de los padecimientos de este síndrome es la enfermedad celíaca (3\% al 7\%) de las personas con síndrome de Down mientras que la prevalencia en la población general se estima que es de 1 por cada 2000 nacidos vivos. La celiaquía se presenta en forma silente, asintomática o atípica, pasando desapercibida en niños con síndrome de Down. Por esta razón, se recomienda el cribado sistemático mediante la determinación de marcadores serológicos después de los tres años de edad y tomando alimentación que contenga gluten al menos durante un año.

Un resultado inicial negativo de los test serológicos no excluye la probabilidad de desarrollar la enfermedad a lo largo de la vida del paciente. La estrategia más conveniente en ese caso es repetir periódicamente la determinación de anticuerpos antitransglutaminasa cada 2 o 3 años o cuando se presente compatible con la enfermedad celíaca. Otra opción es determinar la presencia de marcadores genéticos HLA DQ2 o DQ8, de estar presentes, se sugiere continuar los controles serológicos cada 2 o 3 años. Si los resultados son negativos, el riesgo es mínimo y no ameritará más controles (Soriano, 2007).

Cabe destacar que los niños con síndrome de Down en quienes la enfermedad celíaca puede manifestarse a cualquier edad y tiene una prevalencia mucho mayor (5 a 15\%) versus la población general 3.3\%. Se manifiesta más comúnmente como una enfermedad silente, con síntomas vagos como constipación, dispepsia, dolor abdominal y flatulencia. Esta condición dificulta y retarda el diagnóstico en estos pacientes que en la población en general.

La sociedad médica de los Estados Unidos recomienda realizar la tamización para la enfermedad celíaca con tGTIgA e IgA total a todos los niños con síndrome de Down a los dos 


\section{Enfermedad celíaca en síndrome de Down}

Vol. 3, núm. 1., (2019)

Victoria Samanta Luna Panimboza; Kristell Solange Ladines Torres; Carlos Emilio Paz Illescas; Isrrael Rubén Rugel Cabrera

años de vida y a los menores de 2 años con sintomatología sugestiva debe realizarse la prueba de anticuerpos antigliadina AGA IgA además de las dos pruebas mencionadas al inicio de esta sección (la presencia de marcadores genéticos HLA DQ2 o DQ8)(Hospital Garrahan, Pediatría, 2010).

Tipos de enfermedad celíacas

- Silente: pacientes con serología y estudio genético HLA DQ2/DQ8 positivos, biopsia intestinal compatible pero sin síntomas compatibles.

- Latente: pacientes con estudio genético HLA DQ2/DQ8 compatible pero sin enteropatía y que en algún momento de su vida han manifestado enteropatía dependiente del consumo de gluten. Estos pacientes pueden tener o no síntomas y/o serología compatible,

- Potencial: pacientes con serología y estudio genético HLA DQ2/DQ8 compatibles pero sin lesión en la mucosa intestinal obtenida por biopsia, estos pacientes pueden tener o no síntomas y/o desarrollar enteropatía en el futuro.

\section{Diagnostico}

La historia clínica y el examen clínico constituyen la base para orientar el diagnostico en relación a la atención primaria, posteriormente, se sustenta en el conocimiento de los distintos patrones de síntomas que se presenta la enfermedad, las formas atípicas deben ser considerada. También existen grupos de riesgo debido a la existencia de una enfermedad y/o condición que debe considerar el médico tratante o médico de atención primaria (Polanco, 2011, pág. 2) 


\title{
Enfermedad celíaca en síndrome de Down
}

Vol. 3, núm. 1., (2019)

Victoria Samanta Luna Panimboza; Kristell Solange Ladines Torres; Carlos Emilio Paz Illescas;

Isrrael Rubén Rugel Cabrera

Tabla $N^{\circ}$ 2. Síntomas, signos y alteraciones analíticas que obligan a considerar el diagnóstico

\author{
de la enfermedad celíaca
}

\begin{tabular}{|c|c|c|}
\hline Clínica & Síntomas & Signos y alteraciones \\
\hline 绐昜 & $\begin{array}{l}\text { Diarrea crónica, falta de apetito, } \\
\text { vómitos, dolor abdominal } \\
\text { recurrente, laxitud, irritabilidad, } \\
\text { apatía, introversión, tristeza }\end{array}$ & $\begin{array}{l}\text { Malnutrición, distención } \\
\text { abdominal, hipotrofia muscular, } \\
\text { retraso ponderoestatural, } \\
\text { anemia ferropénica, } \\
\text { hipoprotenemia. }\end{array}$ \\
\hline 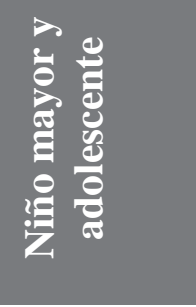 & $\begin{array}{l}\text { Frecuentemente asintomático, } \\
\text { estreñimiento, dolor abdominal, } \\
\text { menarquia retrasada, } \\
\text { irregularidades menstruales, } \\
\text { cefaleas, artralgias, habito de } \\
\text { intestino irregular. }\end{array}$ & $\begin{array}{l}\text { Anemia ferropénica, talla baja, } \\
\text { aftas orales, hipoplasia del } \\
\text { esmalte, distención abdominal, } \\
\text { debilidad muscular, artritis, } \\
\text { osteopenia, queratosis folicular. }\end{array}$ \\
\hline$\frac{\stackrel{\theta}{E}}{\mathrm{E}}$ & $\begin{array}{l}\text { Diarrea crónica, dispepsia, dolor } \\
\text { abdominal recidivante, pérdida de } \\
\text { peso, síntomas de intestino } \\
\text { irritable, vómitos, estreñimiento, } \\
\text { dolores óseos y articulares, } \\
\text { infertilidad, abortos recurrentes, } \\
\text { irritabilidad, astenia, ansiedad, } \\
\text { depresión, epilepsia, ataxia }\end{array}$ & $\begin{array}{l}\text { Malnutrición con o sin pérdida } \\
\text { de peso, edemas periféricos, } \\
\text { talla baja, neuropatía periférica, } \\
\text { miopatía proximal, anemia, } \\
\text { hipoesplenismo, osteopenia, } \\
\text { aftas bucales, descenso de } \\
\text { albúmina sérica, disminución } \\
\text { del tiempo de protrombina, } \\
\text { deficiencia de ácido fólico, } \\
\text { hipertransaminasemia } \\
\text { inexplicada. }\end{array}$ \\
\hline
\end{tabular}

Fuente: I. Polanco. (2011)

Cuando observamos el cuadro $\mathrm{N}^{\circ} 2$ se destaca que muchos signos están presentes en las personas diagnosticadas con síndrome de Down, lo que dificulta aún más su diagnóstico a simple vista. También conviene considerar que en muchos casos el paciente es asintomático, agudizando la salud del paciente. Se recomienda en estos pacientes aplicar una prueba de sangre para 


\section{Enfermedad celíaca en síndrome de Down}

Vol. 3, núm. 1., (2019)

Victoria Samanta Luna Panimboza; Kristell Solange Ladines Torres; Carlos Emilio Paz Illescas; Isrrael Rubén Rugel Cabrera

confirmar el diagnóstico, si la prueba presenta resultados positivos, se recomienda una endoscopia de la vía digestiva alta.

La endoscopia de la vía digestiva alta es un procedimiento que utiliza una cámara flexible y con luz que observa dentro del tracto digestivo, este procedimiento amerita anestesia por un gastroenterólogo. Este especialista de la salud, examinará el intestino y tomará una biopsia para observar si existen signos de enfermedad celíaca.

El diagnóstico se complementa con los hallazgos histológicos de la biopsia del intestino delgado, prueba que es conveniente realizar si existe evidencia de enteropatía sensible al gluten o si se observan anticuerpos endosomiales séricos. El resultado de la biopsia se normaliza dentro de un plazo de 1 a 2 años después de comenzar una dieta sin gluten. La biopsia del intestino delgado pone de manifiesto la lesión difusa de la mucosa en los tramos superiores del intestino delgado que caracteriza a la enfermedad celíaca.

Con un microscopio óptico se observan vellosidades cortas y aplanadas, criptas profundas y un epitelio de superficie irregularmente vacuolado, con linfoncitos en la capa epitelial. Estas anomalías también pueden asociarse a otras afecciones pero improbable de confundir con la enfermedad celíaca.

La respuesta clínica del niño con síndrome de Down diagnosticado con enfermedad celíaca a una dieta sin gluten es positiva, se evidencian cambios una semana posterior al inicio del tratamiento. Mejora el ánimo, disminuye la diarrea, por el contrario, los pacientes con más edad y más enfermos tardan más en reaccionar. 


\section{Enfermedad celíaca en síndrome de Down}

Vol. 3, núm. 1., (2019)

Victoria Samanta Luna Panimboza; Kristell Solange Ladines Torres; Carlos Emilio Paz Illescas;

Isrrael Rubén Rugel Cabrera

\section{Tratamiento}

Establecido el diagnóstico, el régimen terapéutico consiste en una dieta estricta sin gluten que deberá mantener el paciente a lo largo de la vida, obteniendo la desaparición de los síntomas clínicos y la normalización de la mucosa intestinal, se evitan las posibles complicaciones tardías derivadas del consumo del gluten. Se excluirá cualquier producto que contenga trigo, avena, cebada, centeno y todos sus derivados de la dieta del paciente celíaco.

Una dieta $100 \%$ libre de gluten es imposible debido a la contaminación de muchos alimentos pero un consumo menor a $10 \mathrm{mg} / \mathrm{kg}$ al día es seguro. Se define a un alimento como libre de gluten cuando posee menos de $20 \mathrm{mg} / \mathrm{kg}$. Esta dieta mejora y alivia los síntomas, las lesiones endoscópicas e histológicas en la mayoría de los casos. Se alcanza la mejora endoscópica en un 79\% y su normalización en 31\% con un mínimo de 6 meses de dieta. La recuperación histológica se cuantifica en $34 \%$ a los dos años, $66 \%$ a los cinco años y el $90 \%$ a los nueve años luego de iniciada la dieta (Moscoso, 2017).

Otro de los beneficios de la dieta o alimentación libre de gluten, permite el incremento del peso corporal, mejora la densidad mineral ósea y reduce la mortalidad. Se sugiere que un nutricionista acompañe al paciente y su entorno familiar para evitar el consumo de alimentos contaminados y el incremento de peso excesivo en pacientes con síndrome de Down.

Una de las desventajas o retos para cumplir con la dieta libre de gluten es lo costoso de este tipo de alimentos y su poco sabor o no muy atractiva presentación de los alimentos. Este hecho ha motivado nuevas estrategias terapéuticas como ALV003 y AN-PEP, que son proteasas 


\section{Enfermedad celíaca en síndrome de Down}

Vol. 3, núm. 1., (2019)

Victoria Samanta Luna Panimboza; Kristell Solange Ladines Torres; Carlos Emilio Paz Illescas; Isrrael Rubén Rugel Cabrera

que lisan el gluten en el lumen gástrico, evitando la generación de péptidos inmunogénicos que bloquean la apertura del epitelio intestinal disminuyendo su permeabilidad.

Los niveles de anticuerpo anti DGP y anti tTg son dependientes del consumo de gluten y por lo tanto muy convenientes a considerar como indicadores de la evolución y respuesta del paciente a la dieta. El 83\% de los pacientes presenta serología normal luego de 12 meses de dieta libre de gluten, sin embargo un $33 \%$ de los pacientes diagnosticados y en tratamiento, muestra una serología fluctuante, sugiriendo un comportamiento irregular de la dieta(Moscoso, 2017).

La persistencia de serología positiva transcurrido un año de tratamiento, sugiere una ingesta contaminada de alimentos con gluten. La curación de la mucosa podría considerarse el objetivo de la terapia, es importante documentarla con el propósito de mostrar menos complicaciones de salud de los pacientes con síndrome de Down sin que ello signifique eliminar la probabilidad de mortalidad por esta causa.

No siempre la dieta ocasiona una mejora, es el caso de la enfermedad celíaca no colaboradora, se define como la falta de respuesta clínica o de laboratorio luego de 6 a 12 meses de iniciada la dieta libre de gluten. Ocurre entre el $7-30 \%$ de los pacientes y sus causas son exposición al gluten 36\%, síndrome de intestino irritable, enfermedad celíaca refractaria 10\%, intolerancia a la lactosa 8\% y colitis microscópica (Down España, 2012).

La enfermedad celíaca refractaria se define como los signos de malabsorción persistente o recurrentes asociados a atrofias vellositaria a pesar de una dieta estricta libre de gluten por más de 12 meses, en ausencia de otras enfermedades, incluyendo el linfoma. 


\section{Enfermedad celíaca en síndrome de Down}

Vol. 3, núm. 1., (2019)

Victoria Samanta Luna Panimboza; Kristell Solange Ladines Torres; Carlos Emilio Paz Illescas;

Isrrael Rubén Rugel Cabrera

Se destacará lo delicado para el paciente y su entorno familiar de consumir pequeñas cantidades de gluten que puede producir lesiones intestinales aunque no exista repercusión clínica. En general se recomendará evitar el consumo de aquellos alimentos que no tengan información referida a si contiene o no gluten. Los alimentos naturales, no manufacturados y no pertenecientes a la familia de los cereales son seguros: frutas, hortalizas, carnes, pescados, tubérculos, leche, huevos, etc.(González, 2006).

Algunas recomendaciones a considerar a la hora de comprar alimentos preparados y/o envasados que deben leerse en las etiquetas de los productos antes de ser adquiridos e incluidos en la dieta de las personas con intolerancia al gluten con síndrome de Down y en general(Polanco, 2011, pág. 44):

- Los productos alimenticios para personas con intolerancia al gluten no contendrán un nivel de gluten que supere los $100 \mathrm{mg} / \mathrm{kg}$ en los alimentos tal como se venden al consumidor final.

- El etiquetado, la publicidad y la presentación de los productos pueden llevar el término exento de gluten si el contenido no sobrepasa los $20 \mathrm{mg} / \mathrm{kg}$ en total medido en los alimentos listos para ser vendidos al consumidor final.

- La avena contenida en alimentos para personas con intolerancia al gluten debe ser producida o preparada de forma especial para prevenir contaminación con el trigo, centeno o variedades híbridas, cuyo contenido de gluten no debe sobrepasar los 20 $\mathrm{mg} / \mathrm{kg}$. 


\section{Enfermedad celíaca en síndrome de Down}

Vol. 3, núm. 1., (2019)

Victoria Samanta Luna Panimboza; Kristell Solange Ladines Torres; Carlos Emilio Paz Illescas; Isrrael Rubén Rugel Cabrera

- Los términos contenido muy reducido de gluten o exento de gluten deben aparecer muy cerca del nombre comercial del producto para su fácil identificación.

\section{Conclusiones.}

La enfermedad celíaca es un proceso autoinmune y multi sistémico que ocurre en individuos genéticamente predispuestos, consiste en una intolerancia permanente a las proteínas del gluten del trigo, centeno, cebada y del triticale. En su desarrollo contribuyen factores genéticos (HLA, DQ2 Y DQ8), ambientales (gluten) e inmunológico.

El contacto de la mucosa intestinal con el gluten conduce a la aparición de un daño en la mucosa cuyo espectro oscila desde casos con un aumento de la población de linfocitos intraepiteliales hasta formas avanzadas de atrofia vellositaria. Cualquiera de sus formas causa diversos estados carenciales: anemia, osteopenia y un amplio conjunto de síntomas digestivos y extra digestivos.

Las personas con síndrome de Down presentan ciertos riesgos y problemas de salud propios de este síndrome que requieren acciones precisas para garantizar el pleno desarrollo y aprovechamiento de las capacidades de cada persona con este síndrome. El mayor conocimiento de los riesgos asociados a esta condición permite conocer qué alteraciones pueden aparecer y en qué momento o etapa de la vida del paciente, siendo posible añadir un grupo de actividades preventivas así como exploraciones que buscan corregir, aliviar o evitar problemas de salud de estos niños, jóvenes o adultos. 


\section{Enfermedad celíaca en síndrome de Down}

Vol. 3, núm. 1., (2019)

Victoria Samanta Luna Panimboza; Kristell Solange Ladines Torres; Carlos Emilio Paz Illescas;

Isrrael Rubén Rugel Cabrera

Se recomienda realizar la tamización para la enfermedad celíaca con tGTIgA e IgA total a todos los niños con síndrome de Down a los dos años de vida y a los menores de 2 años con sintomatología sugestiva debe realizarse la prueba de anticuerpos antigliadina AGA IgA además de las dos pruebas mencionadas al inicio de esta sección (la presencia de marcadores genéticos HLA DQ2 o DQ8)

Como tratamiento se recomienda seguir el régimen terapéutico de una dieta estricta sin gluten que deberá mantener el paciente a lo largo de la vida, obteniendo la desaparición de los síntomas clínicos y la normalización de la mucosa intestinal, se evitan las posibles complicaciones tardías derivadas del consumo del gluten. Se excluirá cualquier producto que contenga trigo, avena, cebada, centeno y todos sus derivados de la dieta del paciente celíaco. Este tratamiento es para pacientes con síndrome de Down o sin tener esta condición.

Se destacará lo delicado para el paciente y su entorno familiar de consumir pequeñas cantidades de gluten que puede producir lesiones intestinales aunque no exista repercusión clínica. En general se recomendará evitar el consumo de aquellos alimentos que no tengan información referida a si contiene o no gluten, el apoyo de un nutricionista es invaluable en el tratamiento mientras se adecuan a su nuevo estilo de alimentación. Los alimentos naturales, no manufacturados y no pertenecientes a la familia de los cereales son seguros: frutas, hortalizas, carnes, pescados, tubérculos, leche, huevos, etc.

Una de las mayores limitaciones al realizar esta investigación es la poca información estadística de este tema a nivel mundial, incluso la poca existente no está actualizada. El país con mayor información publicada es España, en América Latina esa información es prácticamente 


\section{Enfermedad celíaca en síndrome de Down}

Vol. 3, núm. 1., (2019)

Victoria Samanta Luna Panimboza; Kristell Solange Ladines Torres; Carlos Emilio Paz Illescas; Isrrael Rubén Rugel Cabrera

inexistente, lo cual también es una invitación a los profesionales de la investigación a abocarse a crearla y de ella surjan mejoras en la calidad de vida en quienes padecen esta enfermedad.

\section{Bibliografía.}

Ali, I. (Mayo de 2006). Enfermedad celíaca: formas de presentación clínica en la población pediátrica. Revista de posgrado de la VI Cátedra de Medicina $N^{\circ} 157,2$ - 6. Obtenido de celiaconline.org: www.

Díaz, M. (2012). Enfermedad celíaca: nuevos criterios diagnósticos. Vox Paediátrica XIX (2), 28 -33 .

Down España. (2012). Celíaquia y sindrome de Down: la enfermedad celíaca afecta al 12\% de las personas con síndrome de Down. Sevilla, España.

González, D. (2006). Manejo nutricional de la enfermedad celíaca. Revista Scielo, 207 - 221.

Hospital Garrahan, Pediatría. (26 de Octubre de 2010). Enfermedad celíaca en niños. Buenos Aires, Argentina.

Moscoso, F. (2017). Enfermedad Celíaca. Santa Rosa, Santiago de Chile, Chile.

Polanco, I. (7 de Septiembre de 2011). Estado actual del diagnóstico de la enfermedad celíaca en el niño y adolescente. Madrid, España. Obtenido de dialnet.unirioja.es.

Rodrigo, L. (5 de Marzo de 2008). Enfermedad Celíaca. Oviedo, Asturias, España.

Soriano, J. (Abril de 2007). Actividades preventivas en niños con síndrome de Down. Obtenido de www.ardilladigital.com 